\title{
A D elinquência Juvenil em Perspectiva Comparada: uma Análise Bibliométrica dos Estudos nas Ciências Sociais (2008-2018)
}

\author{
Haydée Caruso* \\ Juliana Ferreira da Silva** \\ Yacine Guellati*** \\ Jairo Castaño López $z^{* * * *}$ \\ Rafael Oliveira ${ }^{* * * * *}$
}

\section{Resumo:}

O artigo apresenta os resultados de análise da produção científica dos últimos 10 anos (2008-2018) acerca da chamada "delinquência juvenil". A análise teve como objetivo compreender quais são as problemáticas associadas às juventudes e às violências e como tais questões têm sido abordadas pela comunidade acadêmica, a partir de estudos empiricamente localizados em países como EUA, França, México, Inglaterra e Brasil. 0 levantamento bibliográfico foi realizado em periódicos nacionais e internacionais de excelência classificados nas áreas da antropologia/arqueologia e sociologia, de acordo com o sistema de avaliação Qualis CAPES. 0 levantamento foi circunscrito aos periódicos disponíveis em língua portuguesa, espanhola, francesa e inglesa que têm como escopo estudos sobre "juventude", "segurança pública" e "violência". Foram sistematizados 289 artigos que por diferentes caminhos teórico-metodológicos abordaram questões relacionadas à imigração, encarceramento em massa, conflitos étnico-raciais, gangues juvenis, tráfico de drogas e violência policial. Palavras-chave: Delinquência juvenil. Violência. Juventude. Segurança pública.

\footnotetext{
* Doutora em Antropologia (UFF). Professora e pesquisadora do Programa de Pós-Graduação em Sociologia da UnB. Bolsista de produtividade em pesquisa 2 do CNPq. Pesquisadora associada do Instituto de Estudos Comparados em Administração de conflitos - INCT-INeAC/UFF. ** Doutora em Psicologia (UFRJ). Professora e Pesquisadora do Programa Pós-Graduação em Psicologia da Universidade Católica de Brasília (UCB). Membro da ABRAPSO e da Sociedade da História da Psicologia da APA. Membro do GT História Social da Psicologia na ANPEPP.

*** Doutorando do Programa de Pós-Graduação em Sociologia (UnB).

**** Doutorando do Programa de Pós-Graduação em Sociologia (UnB).

***** Bacharelando em Sociologia (UnB).
} 


\title{
Juvenile D elinquency in a Comparative Perspective: A Bibliometric Analysis of Studies in Social Sciences (2008-2018)
}

\begin{abstract}
This paper presents the results of an analysis of the scientific production of the last 10 years (2008-2018) about the so-called "youth delinquency". The analysis aimed to understand what are the problems associated to youth and violence and how such issues have been addressed by the academic community, from studies empirically located in countries such as the USA, France, Mexico, England and Brazil. The bibliographic survey was conducted in Brazilian and international journals of excellence classified in the areas of anthropology / archeology and sociology, according to the Qualis CAPES evaluation system. The survey was limited to journals available in Portuguese, Spanish, French and English that focus on studies on youth, law enforcement and violence. A total of 289 articles were systematized that, through different theoretical and methodological approaches, addressed issues related to immigration, mass incarceration, ethnic-racial conflicts, youth gangs, drug trafficking and police violence.
\end{abstract}

Keywords: Youth delinquency. Violence. Youth. Law enforcement.

\section{D elincuencia juvenil en una perspectiva comparada: un análisis bibliométrico de estudios en ciencias sociales (2008-2018)}

Resumen

El artículo presenta los resultados del análisis de la producción científica de los últimos 10 años (2008-2018) sobre la llamada "delincuencia juvenil". El análisis tuvo como objetivo comprender cuáles son los problemas asociados con la juventud y la violencia y cómo la comunidad académica ha abordado dichos problemas, a partir de estudios empíricos ubicados en países como Estados Unidos, Francia, México, Inglaterra y Brasil. La encuesta bibliográfica se realizó en 
revistas de excelencia brasileñas e internacionales clasificadas en las áreas de antropología / arqueología y sociología, de acuerdo con el sistema de evaluación Qualis CAPES. La encuesta se limitó a revistas disponibles en portugués, español, francés e inglés que se centran en estudios sobre la juventud, la seguridad pública y la violencia. Se sistematizaron un total de 289 artículos que, a través de diferentes enfoques teóricos y metodológicos, abordaron cuestiones relacionadas con la inmigración, el encarcelamiento masivo, los conflictos étnico-raciales, las pandillas juveniles, el tráfico de drogas y la violencia policial.

Palabra clave: Delincuencia juvenil. Violencia. Juventud. Seguridad pública.

A análise bibliométrica ora apresentada se constitui no esforço de um coletivo de pesquisadores(as) ${ }^{1}$ que integraram o projeto intitulado "Sociabilidades juvenis na periferia do Distrito Federal: um estudo comparado sobre a relação entre jovens e policiais no Jardim Roriz (Planaltina) e no Sol Nascente (Ceilândia)", desenvolvido no âmbito do Programa de Pós-Graduação em Sociologia da Universidade de Brasília ${ }^{2}$.

A pesquisa visou compreender as sociabilidades juvenis construídas a partir de territórios periféricos do Distrito Federal. Para tanto, optou-se em realizar trabalho de campo sistemático em escolas públicas, com o intuito de, a partir da observação do "mundo da escola", vislumbrar os principais movimentos, circuitos e dinâmicas relacionadas ao mundo dos jovens.

10 grupo de estudos é formado por estudantes de graduação, mestrado e doutorado que sob a orientação da Profa. Haydée Caruso desenvolvem pesquisas relacionadas à cultura policial, relação juventude e polícia, racismo institucional e modelos e policiamento, gangues juvenis, coletivos criminais, entre outros temas. Os autores agradecem às colegas Laura Goncalves de Lima, Gabriela Costa, Natália Adriele Pereira de Sousa e Julia Gasparetto, que contribuíram para realização do levantamento bibliográfico.

2 A pesquisa contou com apoio da Fundação de Amparo à Pesquisa do Distrito Federal (FAP-DF) e foi coordenada pela Profa. Dra. Haydée Caruso (PPGSOL/UnB e INCT-InEAC). 
No contexto brasiliense importou aprofundar o conhecimento sobre o fenômeno das chamadas "gangues juvenis" ${ }^{3}$, todavia, antes do investimento empírico necessário, a equipe se propôs a analisar bibliografias mais recentes acerca de um dos temas clássicos da sociologia, a chamada "delinquência juvenil". 0 desafio de pesquisa estava em primeiro construir "o estado da arte" sobre a questão, através da realização do levantamento bibliográfico que serviu de subsídio para a revisão sistemática e a análise bibliométrica que compõem o material aqui apresentado. Os autores se dedicaram a tematizar as questões que mobilizaram pesquisadores(as) nacionais e internacionais, na última década, em torno da agenda de pesquisa acerca das juventudes e as diversas manifestações de violências.

\section{M ateriais e métodos:}

O levantamento bibliográfico foi realizado no escopo dos artigos acadêmicos publicados no período de janeiro de 2008 a julho de 2018, em periódicos nacionais e internacionais revisados por pares, disponíveis em língua portuguesa, espanhola, francesa e inglesa, classificados como periódicos de excelência acadêmica nas áreas da antropologia/arqueologia e sociologia de acordo com o sistema de avaliação Qualis CAPES/CNPq 4 . 0 levantamento foi circunscrito aos periódicos que têm como escopo estudos sobre "juventude", "segurança pública” e "violência”. Foram uti-

3 Importantes estudos sobre o tema desenvolvidos no DF foram: ABRAMOVAY, M. (org.). Gangues, Gênero e Juventudes: donas de rocha e sujeitos cabulosos. Brasília: Secretaria de Direitos Humanos - SDH, 2010. ABRAMOVAY, M. (et al). Gangues, galeras, chegados e rappers: juventude, violência e cidadania nas cidades da periferia de Brasília. Rio de Janeiro: Garamond, 1999.

4 Foram utilizados os extratos considerados como produção de excelência acadêmica (A1, A2 e B1) no quadriênio 2013-2016. Exceção foi feita para inclusão da revista do Fórum Brasileiro de Segurança Pública, que, embora seja classificada como B5 em sociologia, foi incorporada no levantamento tendo em vista a estreita relação com a temática do projeto. Para mais informações sobre critérios gerais e específicos utilizados em cada área de avaliação da CAPES/CNPq, conferir: https://sucupira.capes.gov.br/sucupira/public/consultas/coleta/coordenadorAreaAvaliacao/listaCoordenadorAreaAvaliacao.jsf 
lizados em português, inglês, francês e espanhol os seguintes termos de busca: gangues, gangues juvenis, galeras, delinquência juvenil, youth gangs, juvenile delinquency, gangs, bandes de jeunes, délinquance juvénile, pandillas juveniles e delincuencia. Por meio da leitura dos resumos, a partir do critério de afinidade temática, foram selecionados 289 artigos. Em seguida, realizou-se a extração de informações bibliométricas do banco de artigos selecionados, as quais foram sistematizados numa planilha do software Excel, que contém: título, resumo curto, link para acessar o artigo, citação completa estilo APA, título do periódico, local do periódico, instituição à qual o periódico está vinculado, autor(a)(es), local onde fica a instituição de pesquisa do(a)(es) autor(a)(es), instituição à qual os(as) pesquisadores(as) estão vinculados(as), local do campo ou referência do estudo e ano da publicação.

\section{Resultados}

Em relação à bibliometria, pode-se afirmar que o volume de pesquisas sobre juventude e delinquência juvenil, considerando critérios e áreas de concentração adotados na seleção, tem oscilado ao longo da última década. Ressalva importante de que o baixo número de publicações em 2018 se deve ao término do levantamento no primeiro semestre de 2018.

Tabela 01 - Artigos publicados no período de 2008 a 2018

\begin{tabular}{|l|l|l|l|l|}
\hline Ano da publicação & n. & & Ano da publicação & n. \\
\hline 2008 & 27 & & 2013 & 30 \\
\hline 2009 & 25 & & 2014 & 22 \\
\hline 2010 & 18 & 2015 & 29 \\
\hline 2011 & 33 & & 2016 & 29 \\
\hline 2012 & 31 & & 2017 & 36 \\
\hline 2013 & 30 & Total & 289 \\
\hline
\end{tabular}


Gráfico 01 - Artigos publicados no período de 2008 a 2018

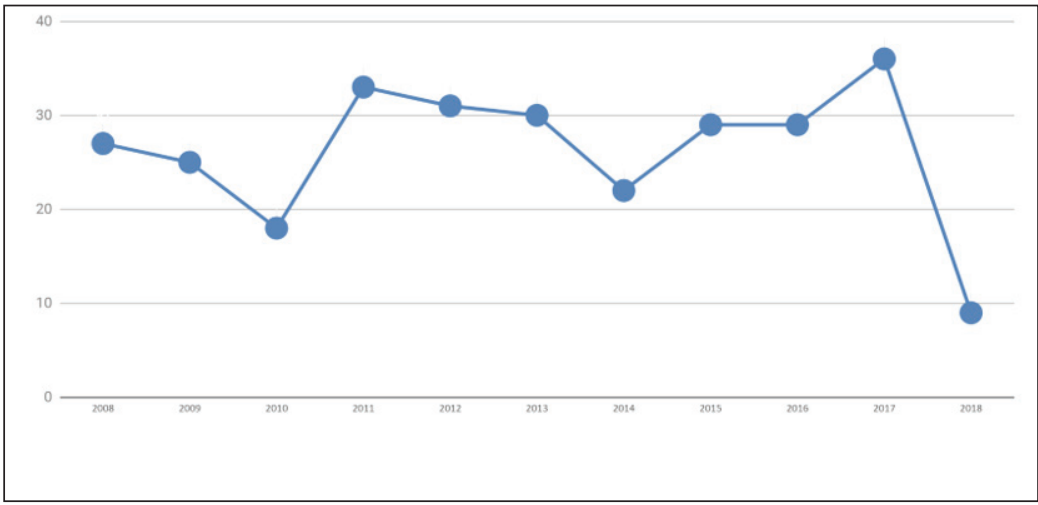

Fonte: elaboração própria (2019)

Quanto à autoria dos artigos, não há predomínio de autores que mais tenham publicado nos últimos 10 anos sobre o tema. 0 volume da produção relacionada à juventude e delinquência juvenil, de acordo com critérios e áreas adotados na seleção, está entre 01 e 02 artigos por autor(a). Não há, portanto, pesquisadores(as) que se destaquem como tendo maior lastro de estudos na temática. No que tange à distribuição geográfica, pode-se notar que as pesquisas são publicadas em sua maioria por periódicos associados a instituições de ensino superior e entidades de pesquisa de seis países, a saber: Inglaterra, Alemanha, Estados Unidos da América (EUA), Brasil, França e México. 
Tabela 02 - Periódicos com pesquisas sobre juventude e delinquência juvenil - Distribuição por país do periódico

\begin{tabular}{|c|c|c|}
\hline País & n. & Periódico \\
\hline Inglaterra & 95 & $\begin{array}{l}\text { British Journal of Sociology of Educacion } \\
\text { Citizenchip Studies } \\
\text { Policing \& Society } \\
\text { Third World Quarterly }\end{array}$ \\
\hline Alemanha & 63 & Crime, Law and Social Change \\
\hline EUA & 40 & $\begin{array}{l}\text { American Anthropologist } \\
\text { Current Anthropology } \\
\text { Current Sociology } \\
\text { Journal of Conflict Resolution } \\
\text { Policy Studies } \\
\text { Research in Social Stratification and Mobility } \\
\end{array}$ \\
\hline Brasil & 28 & $\begin{array}{l}\text { Cadernos PAGU } \\
\text { Ciências Sociais UNISINOS } \\
\text { CIVITAS. Revista de Ciências Sociais } \\
\text { Contexto Internacional } \\
\text { Dados - Revista de Ciências Sociais } \\
\text { Dilemas - Revista de Estudos de Conflito e Controle Social } \\
\text { Educação e Realidade } \\
\text { Horizontes Antropológicos } \\
\text { Revista Brasileira de Ciências Sociais } \\
\text { Revista Brasileira de Segurança Pública } \\
\text { Sociedade e Estado } \\
\text { Sociologias } \\
\text { Tempo Social } \\
\text { Vibrant }\end{array}$ \\
\hline França & 27 & $\begin{array}{l}\text { Cahiers du Genre } \\
\text { Déviance et société } \\
\text { Espaces et sociétés } \\
\text { Hermés } \\
\text { Information sur les sciences sociales/Social Science Infor- } \\
\text { mation } \\
\text { Problèmes d'Amérique Latine } \\
\text { Revue Française de Sociologie }\end{array}$ \\
\hline México & 14 & $\begin{array}{l}\text { Desacatos. Revista de Ciencias Sociales } \\
\text { Estudios Sociologicos } \\
\text { Revista Mexicana de Sociologia }\end{array}$ \\
\hline Holanda & 8 & Contemporary Security Policy \\
\hline Colômbia & 7 & $\begin{array}{l}\text { Revista Latino-Americana de Ciencias Sociales, Niñez y Ju- } \\
\text { ventud }\end{array}$ \\
\hline Portugal & 5 & Sociologia, Problemas e Práticas \\
\hline Espanha & 2 & América Latina Hoy \\
\hline Total & 289 & \\
\hline
\end{tabular}


Gráfico 02 - Periódicos com pesquisas sobre juventude e delinquência juvenil - Distribuição por país do periódico

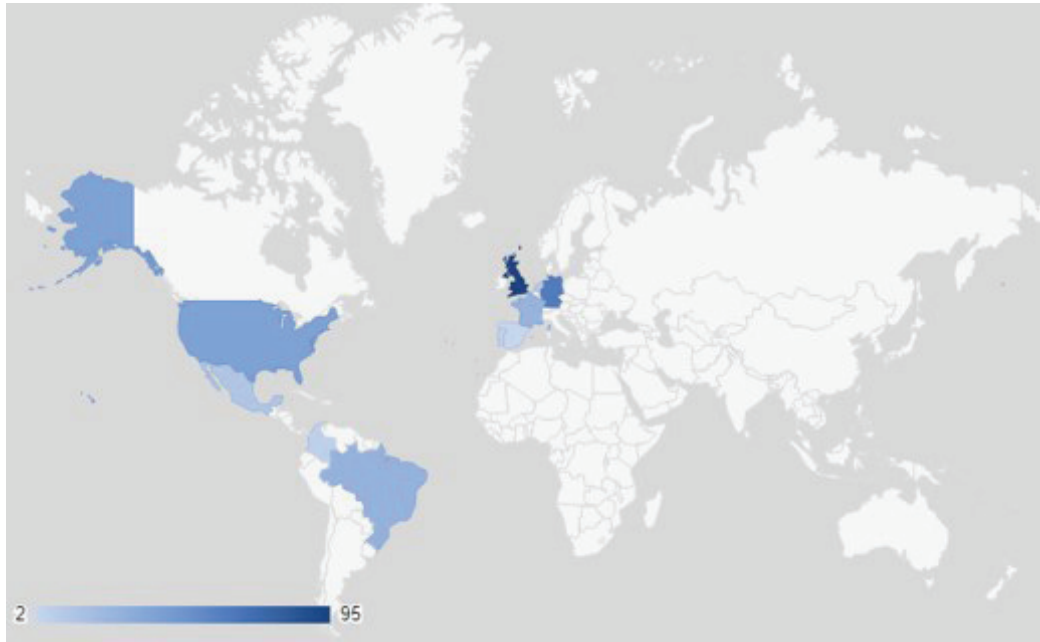

Fonte: Elaboração própria (2019)

As pesquisas têm sido desenvolvidas por instituições ligadas majoritariamente a cinco países, a saber: EUA, Inglaterra, Brasil, Canadá e França. Em sua grande maioria, são também instituições de ensino superior, com exceção da França, onde se encontra em maior número entidades de pesquisa não associadas a universidades. 
Tabela 03 - Pesquisas sobre juventude e delinquência juvenil - Distribuição por nacionalidade da Instituição de Pesquisa

País n. Instituição de Pesquisa

EUA $\quad 73$ Center on International Cooperation, New York University Crimes Against Children Research Center, University of New Hampshire

Department of Anthropology at Johns Hopkins University

Department of Anthropology at the University of Oklahoma

Department of Anthropology, University of Miami

Department of Anthropology, Hofstra University

Department of Behavioral Sciences at Saint Francis University

Department of Communication Studies, Arizona State University

Department of Criminal Justice at California State University San

Bernardino

Department of Criminal Justice at Loyola University

Department of Criminal Justice at University of Cincinnati

Department of Criminal Justice, Temple University

Department of Criminology and Criminal Justice, Indiana University

of Pennsylvania

Department of Criminology University of South Florida

Department of Economics, John Jay College of Criminal Justice at The

City University of New York

Department of Justice, Law and Criminology at American University

Department of Law, Police Science and Criminal Justice, John Jay Col-

lege City University of New York

Department of Political Science, University of Missouri-St. Louis

Department of Political Science and Policy Studies

Department of Political Science at Brooklyn College

Department of Political Science Marquette University

Department of Political Science, Auburn University

Department of Political Science, Northwestern University

Department of Political Science, University of Massachusetts

Department of Political Science, University of New Mexico

Department of political Science, University of North Carolina at Chape Hill

Department of Sociology and Criminal Justice at Villanova University Department of Sociology at Bates College

Department of Sociology at Bowling Green State University

Department of Sociology at the University of Texas

Department of Sociology University of Pittsburgh

Department of Sociology, Criminal Justice, \& Women's Studies, University of South Carolina Upstate

Department of Sociology, Criminology and Anthropology, University of Central Arkansas

Department of Sociology, Purdue University

Department of Sociology, University of New Mexico

Edmund A. Walsh School of Foreign Service, Georgetown University

Emerson College 
Continuação

País n. Instituição de Pesquisa

EUA $\quad 73$ Institute for Policy Research, Northwestern University

Interuniversity Institute for Research and Development (INURED)

Kenan Institute for Ethics, Duke University, Durham

Kimberly Green Latin American and Caribbean Center, Florida

International University

Massachusetts Institute of Technology. Department of Urban Studies and Planning"

Sociology and Anthropology, Northwestern University

Princeton University

Rutgers University

Schar School of Policy and Government, George Mason University

School of Criminal Justice and Criminalistics, California State University

School of Criminology \& Criminal Justice, Arizona State University

School of Criminology and Criminal Justice at Northeastern University

School of Criminology and Criminal Justice, University of Nebraska at Omaha

School of Criminology and Criminal Justice Northeastern University

School of Law, University of California

School of Public Policy University of Maryland

School of Social Science at the Institute for Advanced Study

School of Social Science at the University of California

Seattle Police Department

Social Sciences Division, University of California

State University of New York at Oswego

The Center for Social Medicine at the Semel Institute, University of

California

The Ohio State University

Universidad de Kentucky

University of California

University of Central Florida

University of New Haven

University of Pennsylvania

University of Southern Californ

University of Texas at Austin 
Continuação

País n. Instituição de Pesquisa

Inglaterra 37 African Studies Centre at Oxford University

Cardiff Centre for Crime, Law and Justice, School of Social Sciences, Cardiff University

Centre for Applied Criminology , Birmingham City University

Criminology, Cardiff School of Social Sciences at Cardiff University

Department of Anthropology, Binghamton University

Department of Educational Research at Lancaster University

Department of Information Science, Loughborough University

Department of Law and Criminology, Aberystwyth University

Department of Organization, Work and Technology, Lancaster University

Department of Political Science and Public Policy at the University of Southampton

Department of Social Policy, LSE

Department of Sociology , University of Warwick

Edge Hill University

Institute of Criminology Cambridge University

Institute of Education at London's Global University

Institute of Education at University of Huddersfield

London Metropolitan University

London's Global University

Manchester eResearch Centre, Manchester University

Methodology Institute and Mannheim Centre for Criminology, London School of Economics and Political Science

Roméo Dallaire Child Soldiers Initiative, Dalhousie University

School of Global Studies, University of Sussex

School of Law, King's College London

School of Law, University of Manchester

School of Social \& Health Sciences, University of Abertay Dundee

School of Social and Political Science, Chrystal Macmillan Building

School of Sociology and Criminology at Keele University

School of Sociology, Politics and International Studies, University of Bristol

St Antony's College, School of Interdisciplinary Area Studies, University of Oxford

The Department of Sociology , University of Essex

University of Bradford

University of Edinburgh

University of Glamorgan

University of Sheffield, School of Law

University of Surrey 
País n. Instituição de Pesquisa

Brasil 27 Centro de Estudos de Criminalidade e Segurança Pública (UFMG)

Centro Universitário UNA/BH

Departamento de Ciências Sociais da Universidade Federal de São Paulo Departamento de Sociologia da Universidade Federal do Rio de Janeiro Faculdade de Educação da Universidade Federal do Rio Grande do Sul (UFGRS)

Governo Municipal de Belo Horizonte-MG

Instituto de Estudos Sociais e Políticos (Iesp) da Universidade do Estado do Rio de Janeiro

Instituto de Psicologia da Universidade Federal do Rio Grande do Sul (UFGRS)

Núcleo de Estudos da Violência da Universidade de São Paulo (NEV-USP) Núcleo de Estudos de Políticas Públicas em Direitos Humanos (NEPPDH) da Universidade Federal do Rio de Janeiro (UFRJ)

Observatório das Metrópoles da Universidade Federal do Rio de Janeiro Programa Avançado de Cultura Contemporânea (PACC) da Universidade Federal do Rio de Janeiro

Programa de Estudos Pós-graduados em Psicologia Social da Pontifícia Universidade Católica de São Paulo (PUC-SP)

Programa de Pós-Graduação em Sociologia da Universidade Federal do Rio de Janeiro

Universidade Católica de Brasília

Universidade de São Paulo (USP)

Universidade do Estado do Rio de Janeiro (UERJ)

Universidade Federal de Minas Gerais

Universidade Federal de Pelotas (UFPel)

Universidade Federal do Rio Grande do Sul (UFGRS)

Canadá 23 Carleton University

Centre for Criminology and Sociolegal Studies, University of Toronto Centre International de Criminologie Comparée, School of Criminology, Université de Montréal

Criminology, Justice and Policy Studies at University of OntarioInstitute of Technology

Département de criminologie Université d'Ottawa

Department of History, McGill University

Department of Sociology and Anthropology at Carleton University

Department of Sociology at University of Victoria

Department of Sociology, Social Science Centre, University of Western Ontario

École des hautes études en sciences sociales (EHESS/IRIS); Université du Québec à Montréal

Faculty of Criminology, Justice and Policy Studies at University of Ontario: Institute of Technology

Human Resources and Skills Development

McMaster University

School of Criminology, Université de Montréal

School of Social Sciences Atkinson and Faculty of Graduate Studies

Université Laval, Canada

University of Waterloo 
Continuação

País n. Instituição de Pesquisa

França 23 Centre de recherches sociologiques sur le droit et les institutions pénales, France

Centre Lillois d'études et de recherches sociologiques et économiques (CLERSÉ), Université de Lille 3

Centre National de Recherche Scientifique - CNRS

Cesdip-UVSQ-CNRS-Ministère de la justice-UCP

Department of English and American Studies, University of ParisSorbonne

École des hautes études en sciences sociales (EHESS/IRIS)

Faculté des Sciences économiques et sociales Université Lille 1, França

Institut de recherche interdisciplinaire sur les enjeux sociaux, Université Paris 13 à Bobigny, France

Institut Des Hautes études D’Amérique Latine; Université Paris X Nanterre

Institut Universitaire de France Université de Lille 1

Programme interdisciplinaire de recherche en sciences du sport et du mouvement humain - Sports, organisations, identités (prissmhsoi) de l'Université de Toulouse, França

Université de Cergy-Pontoise

Université J. Monnet Saint-Étienne, França

Université Paris Descartes (Paris V)

Gráfico 03 - Pesquisas sobre juventude e delinquência juvenil - Distribuição por nacionalidade da Instituição de Pesquisa

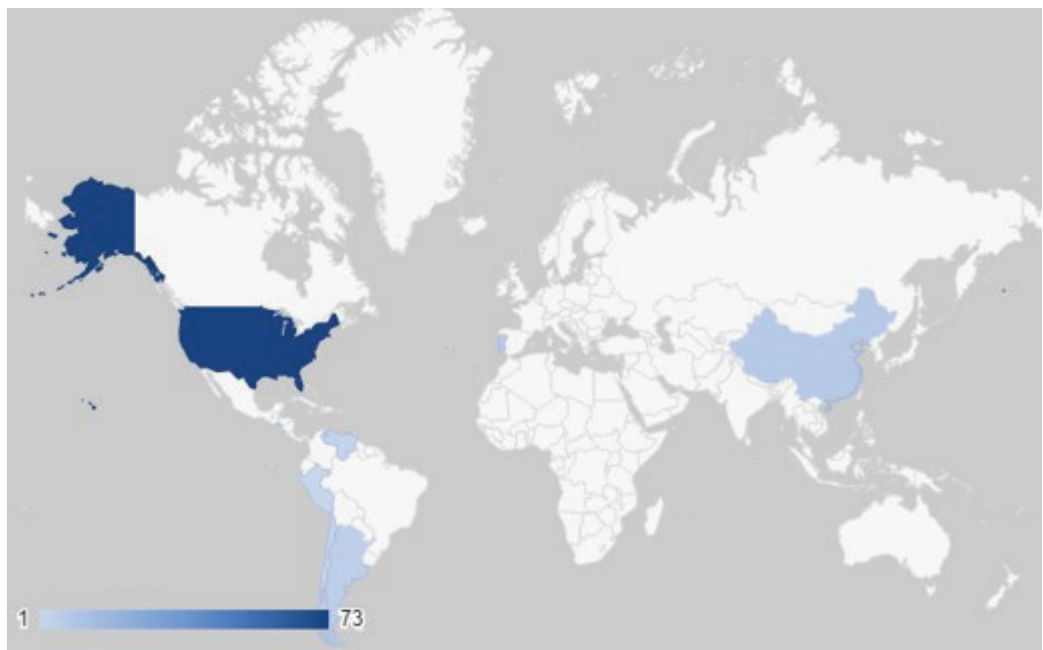

Fonte: Elaboração própria (2019) 
Já quanto ao campo empírico pesquisado, há concentração de estudos nos EUA, Brasil, Inglaterra, França e México. Os dois primeiros países representam majoritariamente os campos empíricos das pesquisas sobre juventude e delinquência juvenil, segundo os critérios de seleção adotados na busca.

Tabela 04 - Campo empírico das pesquisas sobre juventude e delinquência juvenil - distribuição por país

\begin{tabular}{|c|c|c|}
\hline País & n. & Referências \\
\hline EUA & 45 & $\begin{array}{l}\text { Morselli, C. et al, 2016; Mitchell et al, 2010; Levi, 2008; McGuire, } \\
\text { 2013; Briant Carant, 2017; Goldschmidt \& Anonymous, 2008; Papp } \\
\text { et al, 2017; Kim \& Matz, 2018; Moule; Decker; Pyrooz, 2017; Dhondt, } \\
\text { 2012; Rose \& Baumgartner, 2013; Friman, 2009; Hannon \& DeFina, } \\
\text { 2012; Rocque \& Posick, 2017; Mowen \& Manierre, 2017; Skaggs, } \\
\text { 2017; Vuolo, 2014; Nicholls, 2014; Willits \& Nowacki, 2014; Duck } \\
\text { \& Rawls, 2012; DeKeseredy \& Schwartz, 2010; Bouagga, 2010; Cyn- } \\
\text { thia Ghorra-Gobin, 2008; Farrington, 2017; Pickus, 2014; Terwindt, } \\
\text { 2014; Farrell \& Cronin, 2015; Vallas; Zimmerman; Davis, 2009; Gra- } \\
\text { ziano \& Gauthier, 2017; Singer, 2017; Frost, \& Gross, 2012; Nix et } \\
\text { al, 2017; Farrell, Owens, McDevitt, 2014; Martinez, Iwama, Stowell, } \\
\text { 2015; Coutin et al 2017; Reuter, 2009; Montoya, 2013; Kerlikowske, } \\
\text { 2008; Wang \& Zhao, 2016; Schildkraut, Elsass, Stafford, 2015; Bour- } \\
\text { gois, 2018; Painter II, 2010; Lehman-Frisch, 2008; Kubrin, 2017; } \\
\text { Bühler-Niederberger, 2010 }\end{array}$ \\
\hline Brasil & 37 & $\begin{array}{l}\text { Delarre, Melo, Silva, 2015; Doob, 2008; Mendoza, 2015; Souza } \\
\text { \& Souza, 2010; Rocha, 2015; Marinho \& Vargas, 2015; Alvarado, } \\
\text { 2013; Silva, 2011; Paula, 2015; Misse, 2008; Bessire, 2014; Lacer- } \\
\text { da, 2016; Wolff, 2017; Schultze-Kraft, Chinchilla, Moriconi, 2018; } \\
\text { Chétry, 2013; Mattos, 2012; Conte, 2008; Alves \& Arias, 2012; } \\
\text { Waddington et al, 2009; Vinuto \& Alvarez, 2018; Zaluar, } 2012 \text { and } \\
\text { 2014; Biehl \& Locke, 2010; Souza, 2012; Vicentin, 2011; Arias \& } \\
\text { Barnes, 2017; Zubillaga et al, 2008; Gomes et al, 2013; Sento-Sé } \\
\text { \& Coelho, 2014; Zilli \& Beato, 2015; Beato \& Zilli, 2012; Bugnon \& } \\
\text { Duprez, 2014; Bühler-Niederberger, } 2010\end{array}$ \\
\hline $\begin{array}{l}\text { Inglater- } \\
\text { ra }\end{array}$ & 26 & $\begin{array}{l}\text { Williams et al, 2013; Newburn, 2018; King, 2013; Levi, 2008; Body- } \\
\text { Gendrot, 2013; Cooke \& Sturges, 2009; Jennings et al, 2017; Hart, } \\
\text { 2009; Béal, Charvolin, \& Journel, 2011; Henshall, 2018; Coomber, } \\
\text { Moyle, Mahoney, 2019; Fletcher, Bonell, Rhodes, 2009; Fisher \& } \\
\text { Fisher, 2009; Procter et al, 2013; Waddington, et al, 2009; Parmar, } \\
\text { 2011; Medina Ariza, 2014; Griffiths, 2017; Bailey, 2013; Faas, 2008; } \\
\text { Mac Ginty \& Richmond, 2013; Maher \& Pierpoint, 2011; Clayman \& } \\
\text { Skinns, 2012; Moran-Ellis, 2010; Bühler-Niederberger, } 2010\end{array}$ \\
\hline
\end{tabular}




\begin{tabular}{|l|l|l|}
\hline País & n. & Referências \\
\hline França & 18 & $\begin{array}{l}\text { Rocha Vasconcelos, 2015; Lequesne, 2016; Mucchielli, 2008; Zau- } \\
\text { berman } \text { et al, 2013; Zauberman et al, 2013; Mohammed, 2009; } \\
\text { Chéronnet, 2015; Marinho \& Vargas, 2015; Mauger, 2016; de } \\
\text { Maillard et al, 2018; Body-Gendrot, 2013; Damico \& Meyer, 2010; } \\
\text { Joannin \& Mennesson, 2014; Quéro \& Dupont, 2019; Zilli \& Beato, } \\
\text { 2015; Sirota, 2010; Bühler-Niederberger, 2010 }\end{array}$ \\
\hline México & 17 & $\begin{array}{l}\text { Frühling, 2012; Bergman, 2013; Mendoza, 2015; Guerra, 2018; Al- } \\
\text { varado, 2013; Kron, 2016; Durán-Martínez, 2015; Schultze-Kraft, } \\
\text { Chinchilla, Moriconi, 2018; Pérez Correa, 2013; Cruz Sierra, 2014; } \\
\text { Tello, 2012; Savenije, 2010; Zepeda Gil, 2018; García Peña, 2009; } \\
\text { Gámaz, Martínez, Córdoba, 2016; Estrada et al, 2009; Müller, 2016 }\end{array}$ \\
\hline
\end{tabular}

Gráfico 04 - Campo empírico dos estudos relacionadas à juventude e delinquência juvenil - distribuição por países

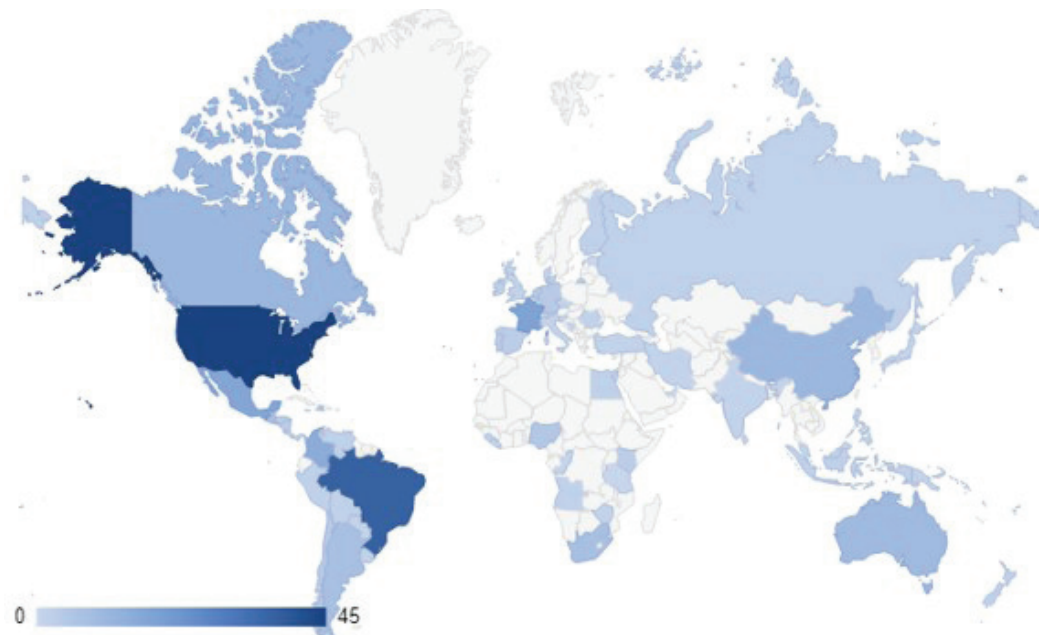

Fonte: Elaboração própria (2019)

\section{D iscussão}

Conforme os dados bibliométricos podem demonstrar, a distribuição por países mostra que as pesquisas estão de múltiplas formas relacionadas principalmente a cinco países. Na Europa se trata da Inglaterra e da França; na América do Sul, o Brasil; na 
América do Norte, os EUA e o México. Tais países estão associados às pesquisas na área por terem mais instituições produzindo pesquisas relacionadas à juventude e delinquência juvenil, por terem mais periódicos publicando tais pesquisas ou por terem seus campos empíricos mais frequentemente pesquisados. Assim, faz-se necessário compreender quais questões contemporâneas afetam a juventude e suscitam a academia na busca por compreensão das dinâmicas da violência e delinquência juvenil.

Inglaterra, França, Brasil, EUA e México são países que demonstram conflitualidades e dinâmicas criminais que possivelmente auxiliem na compreensão do interesse acadêmico sobre o tema da delinquência juvenil. No caso da França e da Inglaterra, questões relacionadas à imigração que têm produzido estudos sobre as conflitualidades envolvendo a juventude. Ambos os países demonstram problemas relacionados à integração social de suas populações de origem imigrante, ainda que tenham desenvolvido políticas sociais inclusivas completamente distintas. Na Inglaterra se tem o modelo integracionista, que reconhece as diferenças culturais, étnicas e religiosas no espaço público e onde as representações dessas comunidades negociam diretamente com o poder público a fim de obter direitos particulares. $\mathrm{Na}$ França se aplica o modelo assimilacionista, que parte do princípio que as populações de origem imigrante, para se integrar a sociedade francesa, devem se "dissolver" na população e assim progressivamente "esconder" tais particularidades culturais, étnicas e religiosas em nome de um republicanismo universalista. Salienta-se que nesses dois países, assim como em outros países da Europa ocidental, a migração de populações das ex-colônias foi requisitada e incentivada por políticas públicas de Estado para auxiliar na reconstrução pós-Segunda Guerra Mundial. Assim, entre anos de 1950 e 1970, tratava-se de uma mão de obra jovem, essencialmente masculina e pouco qualificada, que vinha por motivos de trabalho. Posteriormente, a partir de meados dos anos 1970, no intuito de antecipar os problemas relacionados ao envelhecimento da população, foram incentivadas as mi- 
grações familiares. Assim, a preocupação com a juventude, nesta última década, pode ser compreendida a partir dos processos e dinâmicas sócio-políticas afetos à imigração. (Schnapper, 2003; Noiriel, 2002).

Em ambos os países europeus - França e Inglaterra - jovens imigrantes têm sido alvo de preocupação das autoridades, opinião pública e pesquisadores, por sua suposta participação em crimes de violência letal intencional, crimes contra o patrimônio e vandalismo. A questão imigratória e a estigmatização de jovens imigrantes marcam, nesses dois países, as relações entre policiais e grupos juvenis (Mucchielli, 2008; Mohammed, 2009; Mauger, 2016).

Nota-se também as políticas de policiamento que sofisticam cada vez mais os métodos de controle, ao passo que estigmatizam bairros de maioria de população imigrante (asiáticos, afro-caribenhos, latinos, afrodescendentes e árabes muçulmanos). Contraditoriamente, as pesquisas francesas demonstram que a suposição de que a juventude seria o grande elemento explicativo para a elevação da incidência criminal é falsa, uma vez que a juventude não foi relacionada estatisticamente ao aumento de tais crimes (Mucchielli, 2008; Mauger, 2016). O Estado francês, além disso, é historicamente presente no cotidiano e controle de sua população, o que se pode observar pela perenidade de políticas públicas de planejamento familiar, que datam de mais de dois séculos, nas quais a juventude foi sempre alvo de preocupação.

Já nas Américas do Sul e do Norte, o encarceramento em massa, o tráfico de drogas e a violência letal intencional são as conflitualidades marcantes nas pesquisas relacionadas à delinquência juvenil nestes últimos 10 anos. No período de 2007 a 2017, no Brasil, o encarceramento em massa cresceu na ordem $181 \%$ (Brasil, 2019), sendo marcante o encarceramento de jovens negros pobres e de periferia das grandes metrópoles no país, os 
quais são condenados pela prática de tráfico de drogas e crimes contra o patrimônio. Além disso, o problema dos homicídios constitui a maior preocupação acerca da criminalidade violenta no país, vitimando principalmente esse mesmo público. É coerente, portanto, que as pesquisas brasileiras tenham se dedicado à juventude, buscando compreender os elementos explicativos desses quadros.

No México, a prevalência da juventude e da delinquência juvenil como objeto de pesquisa está relacionada ao acirramento dos contextos de desigualdade, nos quais têm crescido o encarceramento em massa da juventude, cada vez mais vulnerabilizada pelo empobrecimento e pelo desemprego. Ao mesmo tempo, a reconfiguração do mercado de drogas ilícitas trouxe para o México oferta significativa de atividades criminosas, tais como sequestro, extorsão, homicídios e roubo de carga, que se tornaram alternativas de geração de renda ou ingresso em atividades econômicas - ainda que ilegais.

Finalmente, nos EUA, as tensões raciais não resolvidas são o centro das relações conflituosas que integram jovens e as forças policiais. Assim, o problema das violências de gangues e entre gangues e as polícias forma as preocupações relacionadas à delinquência juvenil. Também nesse cenário, marca-se a profunda presença de jovens de minorias étnicas (negros e latinos) como alvo prioritário do sistema de justiça criminal e da justiça juvenil.

\section{Considerações Finais}

A violência praticada pela juventude e a violência que incide sobre a juventude constitui uma questão social que explicita os desafios do enfrentamento social da desigualdade no mundo contemporâneo. Nos artigos brasileiros, a maioria dos trabalhos traz a associação entre delinquência e pobreza como categoria 
de análise preponderante para se pesquisar delinquência, seja de maneira crítica, quando a pobreza não parece ser crucial para se entender a delinquência (Vinuto \& Alvarez, 2018; Paula, 2015; Zaluar 2012; Gurski, 2012); seja como pressuposto (Marinho \& Vargas, 2015; Mattos, 2012; Zilli e Beato, 2012), quando a pobreza se mostra essencial para as dinâmicas de delinquência juvenil do campo.

A categoria "gangue" foi a segunda categoria mais usada como chave explicativa para a delinquência juvenil. No texto de Rocha (2015) a gangue é o que rege as dinâmicas territoriais das "guerras" entre jovens e é dentro da gangue que se estabelecem as sociabilidades entre jovens para alimentar as ditas "rivalidades violentas". Para Zilli \& Beato (2015) as gangues podem, inclusive, ser chave explicativa para entender o aumento nos homicídios no país. Já para Souza \& Souza (2010), as gangues ocupam um lugar de instituição de socialização para os jovens que as constituem, formando "um sentimento de solidariedade e uma vinculação identitária com o grupo" (Souza \& Souza, 2010, p. 281), o que se afasta do texto de Zaluar (2014), que acredita que as gangues trazem apenas vagamente as noções de confiança, lealdade e reciprocidade e "que são as fundações da socialização" (Zaluar, 2014, p. 22).

A última categoria de análise nos estudos sobre Brasil é o contexto educacional. Esta categoria pode aparecer como incidência de violência nas escolas (Souza \& Souza, 2014), impacto da escola e dos métodos de aprendizagem na formação de um estereótipo delinquente (Gomes et al., 2013), dinâmicas internas de socialização e interação nas escolas (Doob, 2006), ou a evasão escolar (Sento-Sé \& Coelho, 2014).

Nas pesquisas sobre o contexto social norte-americano, a temática da delinquência juvenil é um ponto focal nos estudos sobre violência e juventude. Em relação aos adolescentes, Singer (2017) destaca a importância do estabelecimento de relações 
interpessoais entre adolescentes e adultos, assim como adolescentes entre si. 0 autor salienta que tais relacionamentos têm diminuído nos contextos de maior urbanização e crescido em contexto de suburbanização, nos quais relacionamentos entre adolescentes, pais, funcionários de justiça e escola podem ser mais contínuos (Singer, 2017). Destacam-se também nos estudos sobre os EUA, as pesquisas que relacionam a delinquência juvenil e o encarceramento em massa, mostrando que devem ser implementadas reformas nos contextos de justiça juvenil e no sistema de justiça criminal (Hannon \& DeFina, 2012; Duck \& Rawls, 2012). Da mesma forma, discutem-se relações entre as dinâmicas criminais do tráfico de drogas, o acirramento do capitalismo e o encarceramento em massa de jovens em regiões industriais periféricas. Tais contextos econômicos predatórios vulnerabilizam mais grupos empobrecidos que funcionam no varejo de substâncias ilícitas, tornando-os ainda mais marginalizados pelas desigualdades econômicas. Trata-se de uma forma de lucrar com a dinâmica criminal, direcionada à quantidade mínima de capital gerado ilicitamente que é canalizada de volta para a economia lícita, através de multas de justiça criminal, apreensão de ativos por confisco civil e advogados vorazes. De outro lado, o lucro com o encarceramento é ainda obtido dos contribuintes pelos provedores de serviços punitivos e terapêuticos, os quais desfrutam da administração das vítimas da acumulação predatória do capitalismo (Bourgois, 2018).

Cinco artigos sobre o campo empírico francês abordam diretamente a questão da delinquência juvenil (Mucchielli, 2008; Body-Gendrot, 2013; Zauberman et al., 2013a; Zauberman et al., 2013b; Chéronnet, 2015). 0 artigo de Zauberman et al. (2013a) se limita a mencionar que os jovens delinquentes são tanto autores quanto vítimas de violências. Já no outro artigo do mesmo autor (Zauberman, 2013b), este mostra que os medos da população em relação à delinquência juvenil são bastante heterogêneos, mas que as supostas causas são frequentemente relacionadas à perda de moral e à imigração. Mucchielli (2008) aponta 
que a despeito do discurso sobre o aumento das violências no território francês e a suposta relação desta tendência com a delinquência juvenil, as pesquisas mostram que desde os anos 1960 houve uma estabilização geral das violências cometidas por jovens. Sendo assim, tal discurso seria então mais efeito de uma transformação dos valores e do "teto" de tolerância, de uma nova retidão moral frente à violência que tem como desdobramento mais denúncias para a polícia (p. 129). Por fim, Chéronnet (2015) aborda diretamente a questão da delinquência juvenil e se dá por missão desconstruir uma visão homogênea da delinquência, que esta seria produto da opinião pública, e que tem incentivado nos últimos anos os atores da justiça francesa (para menores de idade) a adotar sanções duras ao "isolar os atos cometidos dos elementos da trajetória do jovem" de maneira a mostrar maior serviço para a sociedade (o que ela chama de "maior performance da ação pública"). Por meio de uma análise empírica de reconstituição das trajetórias biográficas de 18 jovens (de 13 a 17 anos e meio de idade), colocando em evidência as dimensões estruturantes de seus percursos delituosos, a autora construiu três categorias ideais-típicas de representação da trajetória delinquente: i) os jovens em renúncia progressiva em relação à carreira delinquente; ii) os jovens que reivindicam sua delinquência como um novo modo de socialização; iii) os jovens que encaram a entrada no percurso delinquente como uma inversão do estigma a eles atrelado. Em suma, para Chéronnet, a trajetória delinquente é mais o "produto de interações sociais e de pontos marcantes que conduzem os jovens a reinterpretar o seu percurso e a dar um sentido a este" (p. 315).

No México, a temática da delinquência juvenil é abordada, principalmente, a partir da desigualdade social como determinante principal da vinculação dos jovens com o mundo criminal, um exemplo desta associação pode ser encontrado num estudo de Bergman (2013) que mostra a correlação positiva entre a inserção precária da juventude mexicana no mundo do trabalho e a vinculação com esquemas de crimes patrimoniais como roubos a 
residências, automóveis e pessoas. Com respeito à problemática do encarceramento da população jovem, a partir das estatísticas oficiais, o estudo de Perez (2014) mostra a grande homogeneidade sóciodemográfica da população carcerária e seu aumento nos anos recentes, neste sentido, se sugere a existência de uma política de estado de encarceramento massivo de jovens de bairros populares. Com respeito à delinquência juvenil vinculada aos crimes contra o patrimônio e aos impactos na saúde, o estudo de Estrada et al. (2009) e o estudo de Zepeda (2018) vão analisar as dinâmicas cada vez mais cruéis da violência juvenil que envolvem assassinato de jovens para demonstrar que no México vem aumentando estas formas de violência por conflitos associados ao tráfico de drogas e que afetam principalmente os jovens.

Na Inglaterra são poucos os estudos que focam na questão da delinquência juvenil, porém, encontramos a pesquisa de Coomber et al. (2019) que descreve formas de policiamento voltadas para grandes "operações contra o crime" que possuem como foco principal jovens (homens e mulheres) que atuam nos postos mais baixos do mercado do crack nas ruas das cidades inglesas. Outro estudo aponta que no imaginário social inglês contemporâneo e no discurso público contra a imigração e favorável à deportação aparecem uma nova figura do "demônio criminoso", o "folk-devil", representado pelo homem jovem migrante ou pertencente a alguma minoria étnica-racial (Griffiths et al., 2017). Finalmente, encontra-se alta participação da população jovem no cyber-crime, identificado como um tipo de crime de "colarinho branco" (White collar) (Levi, 2018).

Por fim, vale destacar que a análise bibliométrica proposta neste artigo visou tão somente permitir ao leitor(a) interessado(a) em temas relacionados às sociabilidades juvenis, de modo geral, e a delinquência juvenil, em particular, encontrar pistas teórico-metodológicas com base em pesquisas empíricas realizadas em contextos geográficos e sócio-políticos dos mais diversos como aqueles aqui apresentados. 


\section{Ref erências}

Alvarado, A. (2013). La violencia juvenil en América Latina. Estudios Sociológicos, 31(91), 229-258. Retrieved from http://www.jstor.org/stable/23622260

Alves, M. C., \& Arias, E. D. (2012). Understanding the Fica Vivo programme: two-tiered community policing in Belo Horizonte, Brazil. Policing and Society, 22(1), 101-113.

Arias, E. D., \& Barnes, N. (2017). Crime and plural orders in Rio de Janeiro, Brazil. Current Sociology, 65(3), 448-465. https://doi. org/10.1177/0011392116667165

Bailey, M. (2013). 'A broadcasting university': educated citizenship and civil prudence. In Governing Through Pedagogy (pp. 75-90). Routledge.

Béal, V., Charvolin, F., \& Journel, C. M. (2011). La ville durable au risque des écoquartiers. Espaces et sociétés, (4), 77-97.

Beato, C., \& Zilli, L. F.. (2012). A estruturação de atividades criminosas: um estudo de caso. Revista Brasileira de Ciências Sociais, 27(80), 71-88. https:// dx.doi.org/10.1590/S0102-69092012000300005

Bergman, M. (2013). Delito patrimonial e inserción laboral en México. Estudios sociológicos, 27-58.

Body-Gendrot, S. (2013). Urban violence in France and England: comparing Paris (2005) and London (2011). Policing and society, 23(1), 6-25.

Bouagga, Y. (2010). Qui sont les «pires des pires»?. Déviance et société, 34(2), 201-216.

Bourgois, P. (2018). Decolonising drug studies in an era of predatory accumulation. Third world quarterly, 39(2), 385-398.

Brasil (2019) Levantamento Nacional de Informações Penitenciárias Atualização - Junho de 2017 Brasília: Ministério da Justiça e Segurança Pública, Departamento Penitenciário Nacional.

Briant Carant, J. (2017). Unheard voices: a critical discourse analysis of the Millennium Development Goals' evolution into the Sustainable Development Goals. Third World Quarterly, 38(1), 16-41.

Bugnon, G., \& Duprez, D. (2014). Les rapports entre jeunes délinquants et police au Brésil au prisme des logiques pénales, policières et territoriales. Déviance et société, 38(3), 311-337. 
Bühler-Niederberger, D. (2010). Childhood Sociology in Ten Countries: Current Outcomes and Future Directions. Current Sociology, 58(2), 369-384. https://doi.org/10.1177/0011392109354250

Chantraine, G., \& Sallée, N. (2013). Éduquer et punir. Revue française de sociologie, 54(3), 437-464.

Chéronnet, H. (2015). Performance de l'action publique et carrières de jeunes délinquants. Dilemas - Revista de Estudos de Conflito e Controle Social, 0, 299-326.

Chétry, M. (2013). La domination territoriale des favelas par les trafiquants de drogue à Rio de Janeiro. Espaces et sociétés, 155(4), 79-93. doi:10.3917/ esp.155.0079.

Clayman, S., \& Skinns, L. (2012). To snitch or not to snitch? An exploratory study of the factors influencing whether young people actively cooperate with the police. Policing and society, 22(4), 460-480.

Conte, M. (2008). Desafios na Juventude: drogas, consumismo e violências. Educação \& Realidade, 33(2).

Cooke, L., \& Sturges, P. (2009). Police and media relations in an era of freedom of information. Policing \& Society, 19(4), 406-424.

Coomber, R., Moyle, L., \& Mahoney, M. K. (2019). Symbolic policing: situating targeted police operations/'crackdowns' on street-level drug markets. Policing and society, 29(1), 1-17.

Coutin, S. B. et al. (2017) Deferred action and the discretionary state: migration, precarity and resistance. Citizenship Studies, 21.8: 951-968.

Cruz Sierra, S. (2014). Violencia y jóvenes: pandilla e identidad masculina en Ciudad Juárez. Revista Mexicana de Sociología, 76 (4), 613-637.

Cynthia Ghorra-Gobin, "L'entrée des Latinos sur la scène métropolitaine américaine : une ambivalence marquée par des tensions politiques et une influence certaine sur le désir de centralité ", Cahiers des Amériques latines [Online], 59 | 2008, posto online no dia 31 janeiro 2013, consultado o 09 abril 2019. URL : http://journals.openedition.org/cal/1164 ; DOI : 10.4000/ cal.1164

Damico, J. G. S. \& Meyer, D. E. E. (2010). Constituição de masculinidades juvenis em contextos "difíceis": vivências de jovens de periferia na França. Cadernos Pagu, (34), 143-178. https://dx.doi.org/10.1590/S010483332010000100007

de Maillard, J., Hunold, D., Roché, S., \& Oberwittler, D. (2018). Different styles of policing: discretionary power in street controls by the public police in France and Germany. Policing and society, 28(2), 175-188. 
DeKeseredy, W. S., \& Schwartz, M. D. (2010). Friedman economic policies, social exclusion, and crime: Toward a gendered left realist subcultural theory. Crime, Law and Social Change, 54(2), 159-170.

Delarre, S., Melo, A., \& Silva, G. (2015). L'Ancrage géographique de la délinquance des adolescents infracteurs à Belo Horizonte. Dilemas - Revista de Estudos de Conflito e Controle Social, 0, 111-138. Recuperado de https://revistas. ufrj.br/index.php/dilemas/article/view/7318/5897

Dhondt, G. (2012). The bluntness of incarceration: Crime and punishment in Tallahassee neighborhoods, 1995 to 2002. Crime, law and social change, 57(5), 521538.

Doob, A. N. (2006). Prevenindo a delinqüência violenta nos jovens. Revista de Ciências Sociais 37(1).

Duck, W., \& Rawls, A. W. (2012). Interaction orders of drug dealing spaces: Local orders of sensemaking in a poor black American place. Crime, law and social change, $57(1), 33-75$.

Durán-Martínez, A. (2015). To kill and tell? State power, criminal competition, and drug violence. Journal of Conflict Resolution, 59(8), 1377-1402.

Estrada, J. G. S., López, T. M. T., Quinteros, C. R., Villaseñor, N. S. F., \& Abundiz, S. V. (2009). Perspectiva psicosocial en adolescentes acusados por delitos contra la salud y robo. Revista Latinoamericana de Ciencias Sociales, Niñez y Juventud, 7(2), 1491-1512.

Faas, D. (2008). Constructing identities: The ethno-national and nationalistic identities of white and Turkish students in two English secondary schools. British Journal of Sociology of Education, 29(1), 37-48.

Farrell, A., \& Cronin, S. (2015). Policing prostitution in an era of human trafficking enforcement. Crime, Law and Social Change, 64(4-5), 211-228.

Farrell, A., Owens, C., \& McDevitt, J. (2014). New laws but few cases: Understanding the challenges to the investigation and prosecution of human trafficking cases. Crime, Law and Social Change, 61(2), 139-168.

Farrington, D. P. (2017). How and why do Amherst and Newark differ in crime rates? Comments on "America's Safest City". Crime, Law and Social Change, 67(5), 499-503.

Fisher, P., \& Fisher, R. (2009). Tomorrow we live: fascist visions of education in 1930s Britain. British journal of sociology of education, 30(1), 71-82.

Fletcher, A., Bonell, C., \& Rhodes, T. (2009). New counter-school cultures: female students' drug use at a high-achieving secondary school. British Journal of Sociology of Education, 30(5), 549-562. 
Freitas, A. C. V. Campos de possibilidade, liminaridade e deriva: Trajetórias de adolescentes em conflito com a lei na Grande Vitória. Dilemas - Revista de Estudos de Conflito e Controle Social, [S.l.], v. 12, n. 1, p. 170-194, jan. 2019. ISSN 2178-2792. Disponível em: <https://revistas.ufrj.br/index.php/dilemas/ article/view/16858>. Acesso em: 22 abr. 2019.

Friman, H. R. (2009). Drug markets and the selective use of violence. Crime, law and social change, 52(3), 285-295.

Frost, N. A., \& Gross, L. A. (2012). Coercive mobility and the impact of prisoncycling on communities. Crime, law and social change, 57(5), 459-474.

Frühling, H. (2012). A realistic look at Latin American community policing programmes. Policing and society, 22(1), 76-88.

Gámaz, A. B. P., Martínez, N. V. F., \& Córdoba, S. F. (2016). Jóvenes en los intersticios de la precariedad, exclusión y violencia. Ciências Sociais Unisinos, 52(3), 396-404.

García Peña, P. L. (2009). De cuerpo amenazante a cuerpo deseado. Paradojas en torno a los varones "echados a perder": el caso de los cholos transnacionales. Desacatos, (30), 59-74.

Ghorra-Gobin,C. (2008) L'entrée des Latinos sur la scène métropolitaine américaine: une ambivalence marquée par des tensions politiques et une influence certaine sur le désir de centralité. Cahiers des Amériques latines [Online] 59.

Goldschmidt, J., \& Anonymous. (2008). The necessity of dishonesty: police deviance,'making the case', and the public good. Policing and Society, 18(2), 113-135.

Gomes, C. A., Lira, A., Caldas, D. B., Lima, D. A., \& Lopes, R. B. (2013). Violências nas escolas: decifrai-me ou vos devoro. Sociologia, Problemas e Práticas, (71), 39-59.

Graziano, L. M., \& Gauthier, J. F. (2017). Examining the racial-ethnic continuum and perceptions of police misconduct. Policing and Society, 1-16.

Greg Downey, "Being Human in Cities: Phenotypic Bias from Urban Niche Construction," Current Anthropology 57, no. S13 (June 2016): S52-S64.

Griffiths, M. Foreign, criminal: a doubly damned modern British folk-devil. Citizenship Studies, 2017, 21.5: 527-546.

Guerra, E. (2018). Organización armada. La dinámica operativa de los grupos de autodefensa tepalcatepenses. Estudios sociológicos, 36(106), 99-124.

Gurski, R. (2012). Violência juvenil e laço social contemporâneo. Educação \& Realidade, 37(1). 
Hannon, L., \& DeFina, R. (2012). Sowing the seeds: how adult incarceration promotes juvenile delinquency. Crime, law and social change, 57(5), 475-491.

Hart, S. The 'problem'with youth: young people, citizenship and the community. Citizenship studies, 2009, 13.6: 641-657.

Henshall, A. (2018). On the school beat: police officers based in English schools. British Journal of Sociology of Education, 39(5), 593-606.

Jennings, W., Farrall, S., Gray, E., \& Hay, C. (2017). Moral panics and punctuated equilibrium in public policy: An analysis of the criminal justice policy agenda in Britain. Policy Studies Journal, 00 (00).

Joannin, D., \& Mennesson, C. (2014). Dans la cour de l'école. Pratiques sportives et modèles de masculinités. Cahiers du genre, (1), 161-184.

João Biehl and Peter Locke, "Deleuze and the Anthropology of Becoming," Current Anthropology 51, no. 3 (June 2010): 317-351.

Kerlikowske, R. G. (2008). Safe at home? Policing the US hometown in a post 9/11 environment. Crime, law and social change, 50(1-2), 47-58.

Kim, B., \& Matz, A. K. (2018). The reality of partnership: formal collaborations between law enforcement and community corrections agencies in Pennsylvania. Policing and Society, 28(8), 947-967.

King, M. (2013). Birmingham revisited-causal differences between the riots of 2011 and 2005?. Policing and society, 23(1), 26-45.

Kron, S. 'Nacimos de la nada': border struggles and maternal politics in Mexico. Citizenship Studies, 2016, 20.5: 579-594.

Kubrin, C. E. (2017). Delinquency and modernity in cyberspace?: Comments on America's Safest City. Crime, Law and Social Change, 67(5), 505-512.

Lacerda, D. S. (2016). The production of spatial hegemony as statecraft: an attempted passive revolution in the favelas of Rio. Third World Quarterly, 37(6), 1083-1101.

Lehman-Frisch, S. (2008). Le Barrio, une figure majeure du quartier aux ÉtatsUnis. Cahiers des Amériques latines, (59), 79-100.

Lequesne, C. (2016). French foreign and security challenges after the Paris terrorist attacks. Contemporary security policy, 37(2), 306-318.

Levi, M. (2008). White-collar, organised and cyber crimes in the media: some contrasts and similarities. Crime, Law and Social Change, 49(5), 365-377.

Lucas Bessire, "The Rise of Indigenous Hypermarginality: Native Culture as a Neoliberal Politics of Life," Current Anthropology 55, no. 3 (June 2014): 276-295. 
Mac Ginty, R., \& Richmond, O. P. (2013). The local turn in peace building: A critical agenda for peace. Third world quarterly, 34(5), 763-783.

Maher, J., \& Pierpoint, H. (2011). Friends, status symbols and weapons: the use of dogs by youth groups and youth gangs. Crime, law and social change, 55(5), 405-420.

Marinho, F., \& Vargas, J. (2015). Permanências e resistências: Legislação, gestão e tratamento da delinquência juvenil no Brasil e na França. Dilemas - Revista de Estudos de Conflito e Controle Social, 0, 267-298. Recuperado de https://revistas.ufrj.br/index.php/dilemas/article/view/7324/5903

Martinez, R., Iwama, J. A., \& Stowell, J. I. (2015). Race, immigration, and homicide in contemporary Europe and the United States: an urban comparison. Crime, Law and Social Change, 64(4-5), 291-304.

Mattos, C. S. Da valentia à neurose: Criminalização das galeras funk, 'paz' e (auto)regulação das condutas nas favelas. Dilemas - Revista de Estudos de Conflito e Controle Social, [S.l.], v. 5, n. 4, p. 653-680, out. 2012. ISSN 21782792. Disponível em: https://revistas.ufrj.br/index.php/dilemas/article/ view/7411/5958>. Acesso em: 22 abr. 2019.

Mauger, G. (2016). "Jovens de regiões desfavorecidas": delinquência, conflito e radicalização islamita. Tempo Social, 28(2), 39-56. https://doi. org/10.11606/0103-2070.ts.2016.110569

McGuire, R. H. (2013). Steel walls and picket fences: Rematerializing the USMexican border in ambos Nogales. American Anthropologist, 115(3), 466-480.

Medina Ariza, J. J. (2014). Police-initiated contacts: Young people, ethnicity, and the 'usual suspects'. Policing and society, 24(2), 208-223.

Mendoza, Arturo Alvarado. (2015). Nociones de justicia, legalidad y legitimidad de las normas entre jóvenes de cinco países de América Latina. Sociedade e Estado, 30(1), 75-97. https://dx.doi.org/10.1590/S0102-69922015000100006

Michael J. Montoya, "Potential Futures for a Healthy City: Community, Knowledge, and Hope for the Sciences of Life," Current Anthropology 54, no. S7 (October 2013): S45-S55.

Misse, M. (2008). Le movimento. Déviance et société, 32(4), 495-506.

Mitchell, K. J., Finkelhor, D., Jones, L. M., \& Wolak, J. (2010). Growth and change in undercover online child exploitation investigations, 2000-2006. Policing \& Society, 20(4), 416-431.

Mohammed, M. (2009). Les affrontements entre bandes: virilité, honneur et réputation. Déviance et société, 33(2), 173-204

Moran-Ellis,J.(2010). Reflectionson theSociology ofChildhoodintheUK.Current Sociology, 58(2), 186-205. https://doi.org/10.1177/0011392109354241 
Morselli, C., Bouchard, M., Zhang, S., Farabee, D., Ouellet, M., \& Easton, S. (2016). It came from the north: assessing the claim of Canada's rising role as a global supplier of synthetic drugs. Crime, Law and Social Change, 66(3), 247-270.

Moule, R. K., Decker, S. H., \& Pyrooz, D. C. (2017). Technology and conflict: Group processes and collective violence in the Internet era. Crime, Law and Social Change, 68(1-2), 47-73.

Mowen, T. J., \& Manierre, M. J. (2017). School security measures and extracurricular participation: An exploratory multi-level analysis. British Journal of Sociology of Education, 38(3), 344-363.

Mucchielli, L. (2008). Une société plus violente?. Déviance et société, 32(2), 115-147.

Müller, M. M. (2016). Penalizing democracy: punitive politics in neoliberal Mexico. Crime, Law and Social Change, 65(3), 227-249.

Newburn, T., Diski, R., Cooper, K., Deacon, R., Burch, A., \& Grant, M. (2018). 'The biggest gang'? Police and people in the 2011 England riots. Policing and Society, 28(2), 205-222.

Nicholls, W. (2014). Between punishment and discipline: comparing strategies to control unauthorized immigration in the United States. Citizenship studies, 18(6-7), 579-599.

Nix, J., Pickett, J. T., Baek, H., \& Alpert, G. P. (2017). Police research, officer surveys, and response rates. Policing and Society, 1-21.

Noiriel, Gérard; Atlas de l'immigration em France. Paris, Autrement, 2002.

Painter II, M. A. (2010). Get a job and keep it! High school employment and adult wealth accumulation. Research in Social Stratification and Mobility, 28(2), 233-249.

Papp, J., Smith, B., Wareham, J., \& Wu, Y. (2017). Fear of retaliation and citizen willingness to cooperate with police. Policing and Society, 1-17.

Parmar, A. (2011). Stop and search in London: counter-terrorist or counterproductive?. Policing and society, 21(4), 369-382.

Paula, L. (2015). Da "questão do menor" à garantia de direitos: discursos e práticas sobre o envolvimento de adolescentes com a criminalidade urbana. Civitas - Revista de Ciências Sociais, 15(1), 27-43. doi: http://dx.doi. org/10.15448/1984-7289.2015.1.16937

Pérez Correa, C. (2013). Marcando al delincuente: estigmatización, castigo y cumplimiento del derecho. Revista Mexicana de Sociología, 75(2). 
Pickus, N. (2014). Laissez-faire and its discontents: US naturalization and integration policy in comparative perspective. Citizenship Studies, 18(2), 160-174.

Procter, R., Crump, J., Karstedt, S., Voss, A., \& Cantijoch, M. (2013). Reading the riots: What were the police doing on Twitter?. Policing and society, 23(4), 413-436.

Quéro, Y. C., \& Dupont, B. (2019). Nodal governance: toward a better understanding of node relationships in local security governance. Policing and Society, 29(3), 283-301.

Reuter, P. (2009). Systemic violence in drug markets. Crime, Law and Social Change, 52(3), 275-284.

Rocha Vasconcelos, Francisco Thiago. (2015). A invenção da violênciaMUCCHIELLI, Laurent. L'invention de la violence: des peurs, des chiffres, des faits. Paris: Fayard, 2011, pp.340.. Sociologias, 17(39), 404-416. https://dx.doi. org/10.1590/15174522-017003931

Rocha, R. L. S. A guerra como forma de relação: Uma análise das rivalidades violentas entre gangues em um aglomerado de Belo Horizonte. Dilemas - Revista de Estudos de Conflito e Controle Social, [S.l.], v. 8, n. 2, p. 277-301, abr. 2015. ISSN 2178-2792. Disponível em: <https://revistas.ufrj.br/index.php/dilemas/article/view/7293 >. Acesso em: 22 abr. 2019.

Rocque, M., \& Posick, C. (2017). From urban to suburban criminology: Understanding crime in America's "safe" cities. Crime, Law and Social Change, 67(5), 481-488.

Rolim, M., Braga, C., \& Winkelmann, F. (2017) POD RS Socioeducativo e a potência da prevenção terciária Revista Brasileira de Segurança Pública, 11 (1), 148-162.

Rose, M., \& Baumgartner, F. R. (2013). Framing the poor: Media coverage and US poverty policy, 1960-2008. Policy Studies Journal, 41(1), 22-53.

Savenije, W. (2010). Les «Maras» ou la transnationalisation des pandillas en Amérique centrale. Problèmes d'Amérique latine, (1), 111-135.

Schnapper, Dominique. La communauté des citoyens. Folio Essais Gallimard, 2003.

Schildkraut, J., Elsass, H. J., \& Stafford, M. C. (2015). Could it happen here? Moral panic, school shootings, and fear of crime among college students. Crime, Law and Social Change, 63(1-2), 91-110.

Schultze-Kraft, M., Chinchilla, F. A., \& Moriconi, M. (2018). New perspectives on crime, violence and insecurity in Latin America. Crime, law and social change, 69(4), 465-473. 
Sento-Sé, J. T. \& Coelho, M. C.. (2014). Sobre errâncias, imprecisões e ambivalências: notas sobre as trajetórias de jovens cariocas e sua relação com o mundo do crime. Horizontes Antropológicos, 20(42), 327-357. Recuperado de https://dx.doi.org/10.1590/s0104-71832014000200013

Silva, J. C. G. (2011). Sounds of youth in the metropolis: the different routes of the Hip Hop movement in the city of São Paulo.. Vibrant: Virtual Brazilian Anthropology, 8(1), 70-94. https://dx.doi.org/10.1590/S180943412011000100003

Singer, S. I. (2017). A local and universal criminology of modernity: a response to comments on America's Safest City. Crime, Law and Social Change, 67(5), 517-523.

Sirota, R. (2010). French Childhood Sociology: An Unusual, Minor Topic or Well-Defined Field? Current Sociology, 58(2), 250-271. https://doi. org/10.1177/0011392109354244

Skaggs, S. L. (2017). Understanding arrest in rural police-juvenile interactions: A factorial designed survey approach. Policing and Society, 1-18.

Souza, D. (2012). Desvio e estetização da violência: Uma abordagem sócio-antropológica acerca da atividade dos pichadores de muros no Rio de Janeiro. Dilemas - Revista de Estudos de Conflito e Controle Social, 5(2), 267-294. Recuperado de https://revistas.ufrj.br/index.php/dilemas/article/view/7329

Souza, R. S. R., \& Souza, Â. M. D. N. (2010). Juventude e violência: novas demandas para a educação e a segurança públicas. Revista Brasileira de Segurança Pública 6, 4(1)

Tello, N. (2012). Police reforms: the voice of police and residents in Mexico City. Policing and Society, 22(1), 14-27.

Terwindt, C. (2014). Protesters as terrorists?. Crime, Law and Social Change, 62(3), 207-234.

Vallas, S. P., Zimmerman, E., \& Davis, S. N. (2009). Enemies of the state? Testing three models of anti-immigrant sentiment. Research in Social Stratification and Mobility, 27(4), 201-217.

Vicentin, M. C. G. (2011). Corpos em rebelião e o sofrimento-resistência: adolescentes em conflito com a lei. Tempo Social, 23(1), 97-113. https://dx.doi. org/10.1590/S0103-20702011000100005

Vinuto, J. \& Alvarez, M. C. (2018). O adolescente em conflito com a lei em relatórios institucionais. Pastas e prontuários do "Complexo do Tatuapé" (Febem, São Paulo/SP, 1990-2006). Tempo Social, 30(1), 233-257. https://dx.doi. org/10.11606/0103-2070.ts.2018.114545 
Vuolo, M. (2014). Incorporating consensus and conflict into the legitimacy of law. Crime, Law and Social Change, 62(2), 155-170.

Waddington, P. A., Adang, O., Baker, D., Birkbeck, C., Feltes, T., Gabaldón, L. G., ... \& Stenning, P. (2009). Singing the same tune? International continuities and discontinuities in how police talk about using force. Crime, law and social change, 52(2), 111-138.

Wang, L., \& Zhao, J. S. (2016). Contemporary police strategies of crime control in US and China: a comparative study. Crime, Law and Social Change, 66(5), 525-537.

Williams, M. L., Edwards, A., Housley, W., Burnap, P., Rana, O., Avis, N., ... \& Sloan, L. (2013). Policing cyber-neighbourhoods: tension monitoring and social media networks. Policing and society, 23(4), 461-481.

Willits, D. W., \& Nowacki, J. S. (2014). Police organisation and deadly force: An examination of variation across large and small cities. Policing and society, 24(1), 63-80.

Wolff, M. J. (2017). Policing and the logics of violence: a comparative analysis of public security reform in Brazil. Policing and society, 27(5), 560-574.

Zaluar, A. (2012). Juventude violenta: processos, retrocessos e novos percursos Jeunesse violente: processus, régression et nouveaux parcours. Dados, 55(2), 327-365. https://dx.doi.org/10.1590/S0011-52582012000200003

Zaluar, A. (2014). Sociability in crime. Culture, form of life or ethos?. Vibrant: Virtual Brazilian Anthropology, 11(2), 12-46. https://dx.doi.org/10.1590/ S1809-43412014000200001

Zauberman, R., Robert, P., Beck, F., \& Névanen, S. (2013a). Mesurer l'implication des jeunes dans la violence. Déviance et société, 37(1), 89-115.

Zauberman, R., Robert, P., Névanen, S., \& Bon, D. (2013b). Victimation et insécurité en Île-de-France. Revue française de sociologie, 54(1), 111-153.

Zepeda Gil, R. (2018). Violencia en Tierra Caliente: desigualdad, desarrollo y escolaridad en la guerra contra el narcotráfico. Estudios Sociológicos, XXXVI (106), 125-159.

Zilli, L., \& Beato, C. (2015). Gangues juvenis, grupos armados e estruturação de atividades criminosas na Região Metropolitana de Belo Horizonte. Dilemas Revista de Estudos de Conflito e Controle Social, 0, 73-110. Recuperado de https://revistas.ufrj.br/index.php/dilemas/article/view/7317

ZUBILlAGA, V., \& QUIÑONES ACOSTA, R., \& ZÚÑIGA, S., \& FERNÁNDEZ, M. (2008). En búsqueda de salidas a la violencia: relatos de reconversión biográfica de hombres jóvenes en Caracas. Revista Mexicana de Sociología, 70 (4), 759-789. 